\title{
Psychosis in bipolar disorder: Does it represent a more "severe" illness?
}

\author{
Cynthia Z Burton $^{1}$ | Kelly A Ryan ${ }^{1}$ | Masoud Kamali ${ }^{1,2}$ | David F Marshall ${ }^{1}$ | \\ Gloria Harrington $^{1}$ | Melvin G McInnis ${ }^{1}$ | Ivy F Tso ${ }^{1}$
}

${ }^{1}$ Department of Psychiatry, University of Michigan, Ann Arbor, MI, USA

${ }^{2}$ Massachusetts General Hospital, Boston, MA, USA

\section{Correspondence}

Cynthia Burton, 4250 Plymouth Road, Ann Arbor, MI, USA

Email: czburton@med.umich.edu

Funding information

National Institutes of Health, Grant/Award Number: 5KL2TR000434; National Institute of Mental Health, Grant/Award Number: K23MH108823; Heinz C. Prechter Bipolar Research Fund; Richard Tam Foundation
Objectives: Although there is a common clinical assumption that bipolar disorder with psychotic features reflects greater severity than bipolar disorder without psychosis, the existing empirical literature is mixed. This study investigated the phenomenology of psychosis as well as demographic, clinical, functional, and neuropsychological features in a large, cross-sectional sample of participants with bipolar disorder divided by history of psychosis.

Methods: In a large single study, 168 affective-only bipolar disorder (BP-A) participants and 213 bipolar disorder with a history of psychosis (BP-P) participants completed a comprehensive clinical diagnostic interview and neuropsychological testing. $t$ tests, chi-square tests, and Bayes factors were used to investigate group differences or lack thereof.

Results: The prevalence of psychosis in this sample (53\%) was similar to published reports. Nearly half of BP-P participants experienced grandiose delusions, and relatively few endorsed "first-rank" hallucinations of running commentary or two or more voices conversing. There were no demographic or neuropsychological differences between groups. BP-A participants experienced greater chronicity of affective symptoms and a greater degree of rapid cycling than BP-P participants; there were no other clinical differences between groups.

Conclusions: Overall, these results contradict the conventional notion that bipolar disorder with psychotic features represents a more severe illness than bipolar disorder without a history of psychosis. The presence of psychosis does not appear to be associated with poorer clinical/functional outcome or suggest a greater degree of neuropsychological impairment; conversely, the absence of psychosis was associated with affective chronicity and rapid cycling. Nosological and treatment implications are discussed.

\section{KEYWORDS}

affective disorder, neuropsychological functioning, serious mental illness

\section{1 | INTRODUCTION}

Psychosis, generally defined as the occurrence of hallucinations or delusions, is a common feature across numerous psychiatric disorders. ${ }^{1-3}$ An exemplar psychotic illness is schizophrenia, which is often associated with chronic psychosis symptoms and poor psychosocial outcome. Psychosis is also an especially prevalent phenotype in bipolar disorder (BP), with greater than half of all individuals diagnosed with BP experiencing psychotic mood episodes in their lifetime. ${ }^{4}$ Consequently, there is a common clinical assumption that BP with psychosis represents a 
more "severe" form of illness than BP without psychosis, and may resemble the clinical and functional deterioration commonly seen in primary psychotic disorders. This notion is supported by the Diagnostic and Statistical Manual of Mental Disorders, ${ }^{5,6}$ where the presence of psychosis automatically changes an otherwise hypomanic episode to a manic episode (and a corresponding bipolar I disorder diagnosis). Overlapping genetic findings between BP and schizophrenia have also contributed to this assumption, ${ }^{7,8}$ although low odds ratios reported limit the conclusiveness of these results. The empirical literature in this area is mixed. Relatively few studies have examined demographic, clinical, and neuropsychological differences among individuals diagnosed with affective-only BP (BP-A) and psychotic BP (BP-P), with few conclusive findings. The question of whether the presence of psychosis in BP truly represents a more "severe" subtype of illness, as is currently assumed, has important nosological and treatment implications. This study investigated the phenomenology of psychotic features as well as clinical, functional, and neuropsychological differences between BP-A and BP-P participants in a large cross-sectional sample.

Regarding demographic, functional, and clinical differences, a 2010 meta-analysis revealed that, compared with BP-A, BP-P participants had more inpatient hospitalizations, younger age of onset, and fewer years of education, although there were no significant differences for age, gender, or duration of illness. ${ }^{9}$ A later study with 199 BP participants found that BP-P participants had a shorter duration of illness, fewer episodes of elevated mood, fewer current depressive symptoms, and lower current functioning scores than BP-A participants. ${ }^{10}$ Other studies have failed to find any differences between BP-A and BP-P participants. For example, Keck et al. ${ }^{11}$ enrolled 352 participants with bipolar I disorder-the largest published study before this present study-and explored a range of variables including age, gender, ethnicity, education, psychosocial support, income, vocational status, illness characteristics like the presence of mixed episodes or rapid cycling, age of treatment initiation, history of suicide attempts, and presence of comorbid disorders, and found no significant differences between BP-P and BP-A groups. They did find that BP-P participants were less likely to have a first-degree relative diagnosed with $B P^{11}$

As for neuropsychological functioning, several studies have compared neuropsychological performance between BP-A and BP-P participants. A 2010 meta-analysis consolidated this literature, comprising 11 published studies including over 700 participants, and reporting a small but significant difference between BP-P individuals and BP-A individuals in global cognition (Cohen's $d=0.22$ ). ${ }^{9}$ BP-P individuals also performed more poorly in four out of six neuropsychological domains, including planning/reasoning ( $d=0.31)$, working memory $(d=0.28)$, verbal memory $(d=0.39)$, and processing speed $(0.20)$; there were no differences in attention or visual memory. ${ }^{9}$ However, a more recent study reported that BP-P participants performed more poorly than BP-A participants on a measure of semantic fluency, although history of psychosis was not associated with poorer performance on measures of verbal learning and memory, working memory, processing speed, response inhibition, or phonetic fluency. ${ }^{10}$ Additional recent findings have supported a lack of neuropsychological differences between those with and without a history of psychosis among first-treatment bipolar I disorder individuals ${ }^{12}$ as well as euthymic outpatients with a range of bipolar disorder diagnoses. ${ }^{13}$

Given the conflicting findings and relatively small effects in the published literature to date, further examination of the presence of psychosis among people diagnosed with BP and whether it is associated with greater clinical, functional (e.g., occupational or social dysfunction), or neuropsychological impairment is warranted. Clarification of whether BP with psychosis indeed reflects a greater degree of severity is an important inquiry in terms of nosology and treatment. For example, as the field of psychiatry moves toward dimensional symptom and functional descriptions rather than categorical classification, it will be helpful to have a better understanding of what features characterize "bipolar-spectrum" or "psychosis-spectrum" illnesses and whether they produce different targets for intervention. Recognizing similarities and differences between BP with and without psychosis is also important for diagnostic classification and prognostic value. This knowledge may inform providers' clinical decision-making; if the presence of psychosis is associated with poorer functioning, for example, providers may wish to target psychotic symptoms separately or more aggressively than mood symptoms. To address these questions, this study investigated the phenomenology of psychosis as well as demographic, clinical, functional, and neuropsychological characteristics among a large cross-sectional sample of participants with BP, divided by history of psychosis.

\section{2 | MATERIALS AND METHODS}

\section{1 | Participants}

Participants were enrolled in the University of Michigan Prechter Longitudinal Study of Bipolar Disorder, an institutional review board (IRBMED) -approved study which broadly aims to assess biological and environmental factors associated with clinical outcome in a large sample of people diagnosed with BP through longitudinal, naturalistic follow-up. Between February 2006 and December 2010, 405 individuals diagnosed with $\mathrm{BP}$ enrolled in the study; 170 were characterized as having "affective only" BP (BP-A), 213 had a history of psychosis (BP-P), and 22 had an uncertain history of psychosis and were excluded from further analyses.

\section{2 | Procedures}

Potential participants were referred by treating clinicians or selfreferral in response to recruitment advertising in clinic or community settings (including the University of Michigan Human Research Recruiting Registry, office bulletin boards, newspapers, websites, and community outreach events). Inclusion criteria were: (i) age $\geq 18$ years, (ii) diagnosis of BP (including Bipolar I Disorder [BP I], Bipolar II Disorder [BP II], and Bipolar Disorder Not Otherwise Specified [BP NOS]), and (iii) willingness to participate in a longitudinal study. Exclusion criteria consisted of: (i) active/current substance dependence, (ii) medical illness associated with depression including, but not limited to, terminal cancers, Cushing's disease, and stroke, (iii) history 
of severe head injury or other neurological injury, and (iv) substantial intellectual impairment (intelligence quotient $[I Q]<70$ ). Following provision of written informed consent, participants completed a comprehensive assessment consisting of a clinical diagnostic interview (Diagnostic Interview for Genetic Studies [DIGS ${ }^{14}$ ), and neuropsychological testing. Participants' diagnoses were confirmed via medical record review if available, and followed by best estimate consensus regarding diagnosis reached by two independent $\mathrm{MD}$ - or PhD-level clinicians. The presence of any psychosis during an affective episode was rated from information collected on the DIGS as 0 (never), 1 (during depression only), 2 (during mania only), 3 (during both), or 9 (uncertain about presence of psychosis). BP participants rated as 0 (never) were included in the BP-A group, and those rated as 1, 2, or 3 were included in the BP-P group. BP-A participants included 94 individuals diagnosed with BP I, 52 individuals diagnosed with BP II, and 22 individuals with BP NOS. The BP-P group was comprised of 200 individuals with BP I, 10 individuals with BP II, and three individuals with BP NOS. Those who had psychosis outside an affective episode were not diagnosed with BP and were not included in this study.

\section{3 | Measures}

The DIGS is a clinical interview designed to assess major mood and psychotic disorders and related conditions; it also includes a detailed assessment of the course and chronology of symptoms and therefore yields an abundance of clinical information. ${ }^{14}$ Specific clinical variables used in the analyses were derived from the DIGS and included history of mixed episodes, history of suicidality, substance abuse chronicity, functional impact of illness, first-degree relative with BP, chronicity of affective disorder, and presence of rapid cycling. Ratings that were classified as "uncertain" were recoded as missing data. In addition to the DIGS, participants completed numerous neuropsychological measures; these variables were subjected to standard data reduction techniques using confirmatory factor analysis and described elsewhere. ${ }^{15}$ The resulting factors included auditory memory, visual memory, fine motor skill, emotion processing, and four domains of executive functioning: verbal fluency and processing speed, processing speed with interference resolution, conceptual reasoning and set-shifting, and inhibitory control. These neuropsychological domains and their constituent measures are summarized in Figure 1.

\section{4 | Analyses}

Participants with BP and no history of psychosis (BP-A) were compared with participants with BP and a history of psychosis (BP-P) via independent-samples $t$ tests or chi-square tests for demographic, clinical, and neuropsychological variables. The original sample included 170 BP-A participants and 213 BP-P participants; these groups were found to significantly differ on duration of illness (BP-A, mean duration 25.2 years [standard deviation $(S D)=13.8$ years] vs BP-P, 22.4 years $[S D=13.0$ years]; $t=2.06 ; d f=379 ; P=.040$ ). To equate the groups on this variable (a potential confounding factor of other clinical and neuropsychological measures), the two BP-A participants with the longest duration of illness (58 and 57 years, respectively) were excluded, which eliminated the significant difference in duration of illness (BP-A, mean duration 24.8 years [SD=13.5 years] vs BP-P, 22.4 years $[S D=13.0$ years]; $t=1.80 ; d f=377 ; P=.073$ ). The results below, therefore, include $168 \mathrm{BP}-\mathrm{A}$ participants and $213 \mathrm{BP}-\mathrm{P}$ participants, for a total of 381 participants diagnosed with BP. For comparison purposes, we also performed the same analyses using the sample including these two participants, and the results were identical and thus are not reported here.

Continuous clinical variables included age of onset, duration of illness (years), and medication load (described below). Ordinal variables (categories with increasing level of severity) were analyzed identically to continuous variables and included history of mixed episodes, history of suicidal thoughts or behavior, history of substance abuse, and functional impact of illness (a global rating of the degree to which psychiatric illness has led to disability or inability to live independently). Total medication load was assessed using a protocol commonly used in the literature and fully described in a previous report; briefly, it involved coding psychiatric medications based on standardized dose equivalents and summing them to create a composite measure. ${ }^{16}$ Medications were also coded and analyzed between groups according to type, including mood stabilizer, antipsychotic, antidepressant, and benzodiazepine. Categorical variables included first-degree relative with $\mathrm{BP}$, chronicity of affective disorder, and presence of rapid cycling. Category labels for each clinical variable can be found in Figure 2.

To characterize the strength of the evidence in favor of the null hypotheses, Bayes factors were calculated for each of the demographic, clinical, and neuropsychological comparisons using the $\mathrm{R}$ package "BayesFactor". ${ }^{17}$ The Bayesian approach considers data using a ratio that contrasts the likelihood of the data fitting under the alternative hypothesis with the likelihood of fitting under the null hypothesis; this ratio is expressed as the Bayes factor. ${ }^{18}$ The distinct advantage of this approach over (or in addition to) traditional frequentist hypothesis testing is that it provides an estimate of the amount of evidence present in the data, facilitating the interpretation of $P$-values that may be approaching the selected cutoff for statistical significance. ${ }^{18}$ For these analyses, interpretation followed accepted guidelines where Bayes factors between 1 and 3 provide anecdotal evidence for the alternative hypothesis, those between 3 and 10 provide substantial evidence, and those between 10 and 30 provide strong evidence; conversely, Bayes factors between 0.33 and 1 provide anecdotal evidence for the null hypothesis, those between 0.10 and 0.33 provide substantial evidence, and those between 0.03 and 0.10 provide strong evidence. ${ }^{18}$

\section{\begin{tabular}{l|l}
3 & RESULTS
\end{tabular}}

\section{1 | Sample characteristics}

Table 1 includes demographic and clinical characteristics of all participants. On average, participants with BP $(n=403)$ were 41 years old with 15 years of education; the majority (65\%) were women. Their mean age of onset was 18 years, with an average duration of illness of 23 years. 


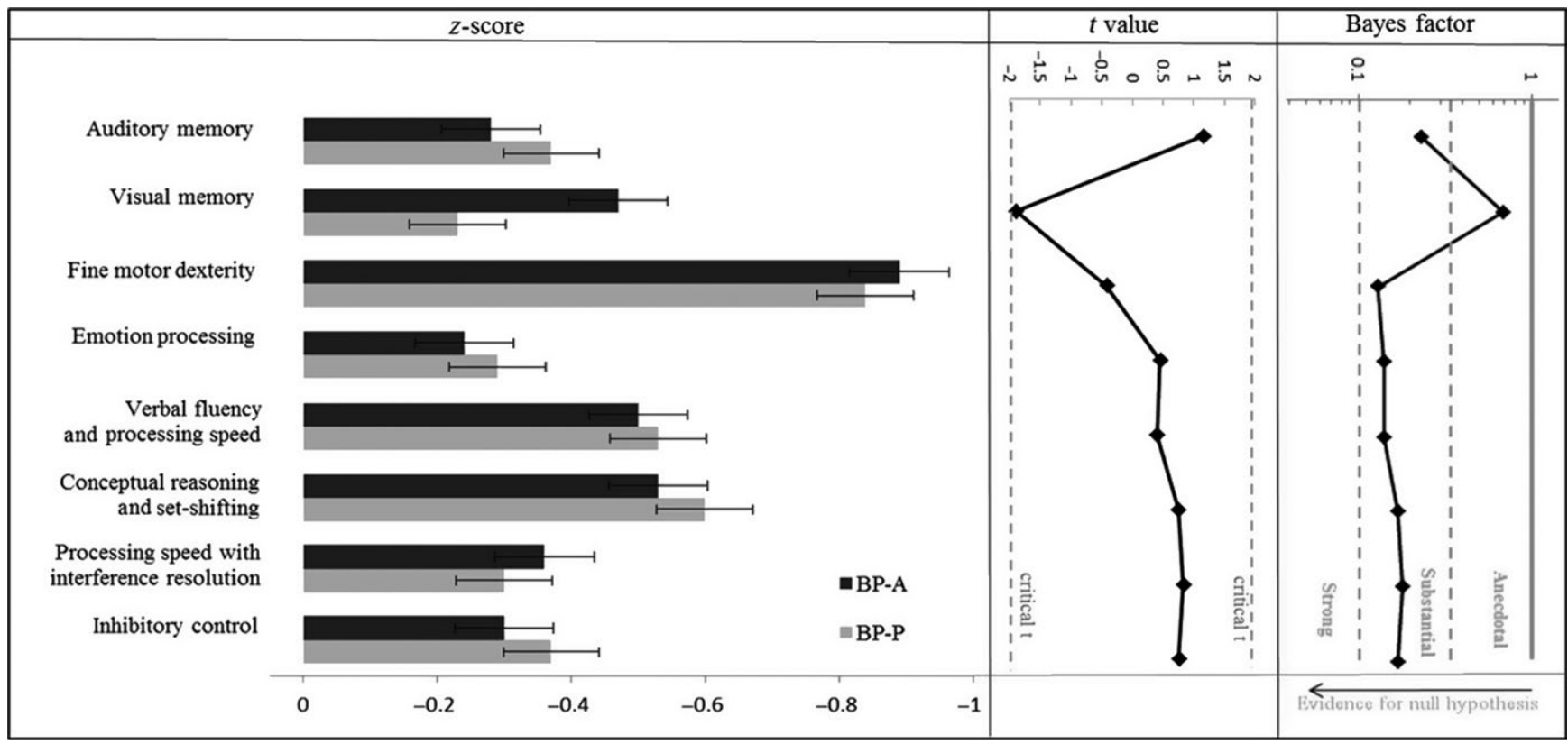

FIGURE 1 Neuropsychological domain z-scores with standard error bars, $t$ statistics, and Bayes factors for group comparisons. These $z$-scores were calculated using the mean and standard deviation of the healthy control (HC) participants enrolled in this study. The $y$-axis on the Bayes factor graph is on a log-scale. The measures comprising each domain included: auditory memory=California Verbal Learning Test, second edition ${ }^{21}$; visual memory=Rey-Osterrieth Complex Figure Test ${ }^{22}$; fine motor dexterity=Purdue Pegboard ${ }^{23}$; emotion processing=Facial Emotion Perception Test ${ }^{24,25}$; verbal fluency and processing speed=Controlled Oral Word Association Test letter and animal fluency, ${ }^{26}$ Wechsler Adult Intelligence Scale, third edition (WAIS-III) Digit Symbol, ${ }^{27}$ Stroop Color-Word Interference Test, color reading and word reading, ${ }^{28}$ and Trail Making Test, part $\mathrm{B}^{29}$; conceptual reasoning and set-shifting=Wisconsin Card Sorting Test ${ }^{30}$ and Parametric Go/No-Go Test mean accuracy ${ }^{31}$; processing speed with interference resolution=Trail Making Test, parts A and B, ${ }^{29}$ WAIS-III Digit Symbol, ${ }^{27}$ Stroop Color-Word Interference Test, interference condition, ${ }^{28}$ and Parametric Go/No-Go Test mean response time ${ }^{31}$; inhibitory control=Parametric Go/No-Go Test mean accuracy and mean response time ${ }^{31}$

\section{2 | Phenomenology of psychosis}

Figure 3 details the range and type of psychotic symptoms reported by participants with a confirmed history of psychosis ( $n=213 ; 53 \%$ ). The majority of BP-P participants reported experiencing delusional ideation, with nearly half experiencing grandiose delusions. Less than half of BP-P participants experienced hallucinations in any modality. Relatively few BP-P participants endorsed traditional "first-rank" hallucinations of running commentary or two or more voices conversing.

\subsection{Comparisons among BP-A and BP-P participants}

Demographically, there were no significant differences between groups on age, education, gender, age of onset, or medication load (all $P>$.152; Table 1). Examining classes of medications, BP-P participants were more likely to be prescribed mood-stabilizing or antipsychotic medication, although Bayes factors indicate only anecdotal evidence for higher rates; there were no differences between groups on prescription of antidepressant or benzodiazepine medication (Table 1).

In terms of clinical and functional variables, there were no significant differences on history of mixed episodes, history of suicidal thoughts or behaviors, chronicity of substance use, functional impact of illness, or first-degree family history of BP (all $P>$.070; Figure 4). The groups significantly differed on chronicity of affective disorder $\left(\chi^{2}=11.34 ; d f=2 ; P=.003\right.$; Figure 4$)$, with a higher proportion of BP-A participants experiencing mood symptoms most of the time compared with BP-P participants. Presence of rapid cycling was also more prevalent among BP-A than BP-P participants $\left(\chi^{2}=7.12 ; d f=2 ; P=.028\right.$; Figure 4). Bayes factors also favored the null hypothesis for most comparisons (Bayes factors ranging from 0.12 to 0.69; Figure 4); the Bayes factor for affective chronicity was 14.35 , suggesting strong support for the hypothesis that the BP-A and BP-P groups differ, and the Bayes factor for presence of rapid cycling was 1.27 , indicating anecdotal evidence that the groups differ (Figure 4).

Regarding neuropsychological functioning, there were no significant differences between BP-A and BP-P participants in any of the eight neuropsychological domains (all $P>$.059; Figure 1 ). Bayes factors ranged from 0.14 to 0.68 , indicating anecdotal to substantial evidence for the null hypothesis (Figure 1).

\section{4 | DISCUSSION}

This study examined the phenomenology of psychosis in BP as well as demographic, clinical, and neuropsychological differences between people diagnosed with BP with and without a history of psychosis. 


\section{(A) History of mixed episodes}

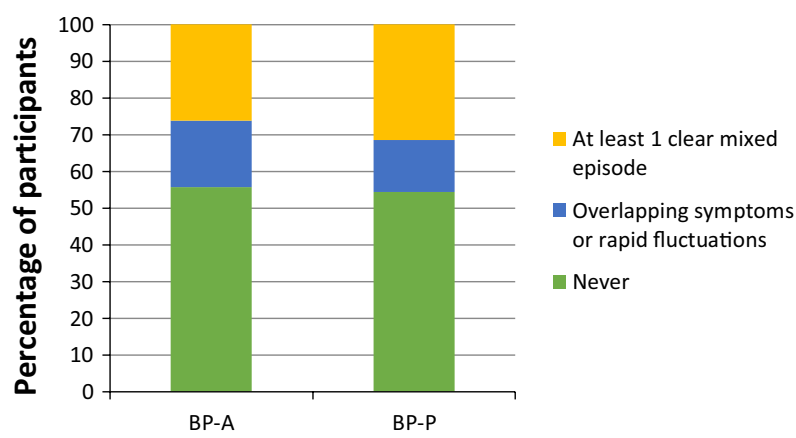

(C) History of substance abuse

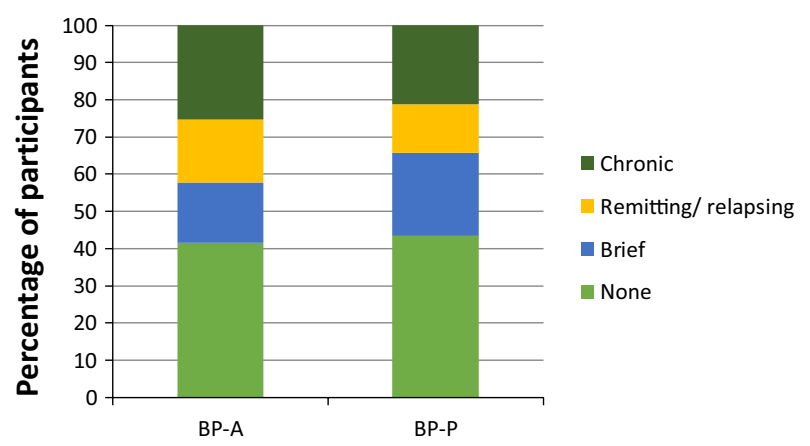

(E) First-degree relative with bipolar disorder

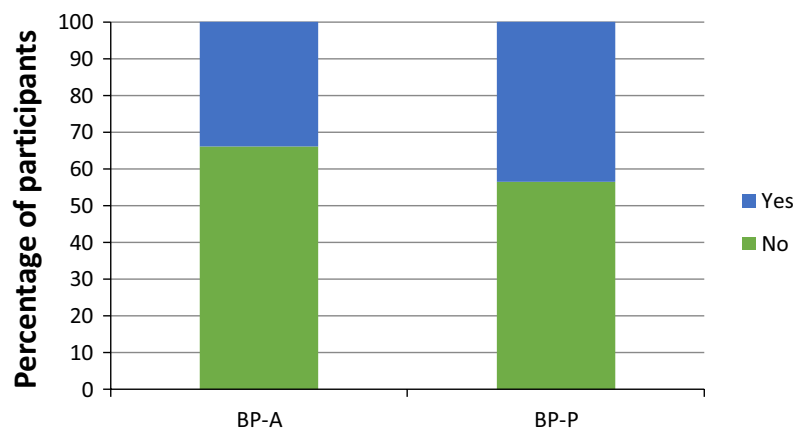

(G) Presence of rapid cycling

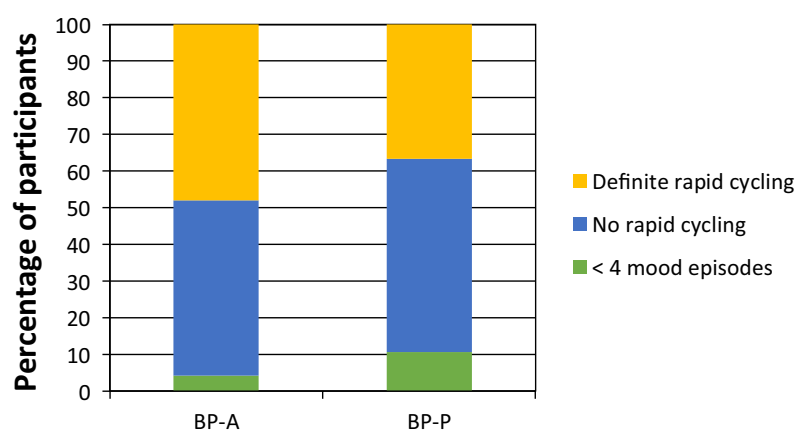

(B) History of suicidality

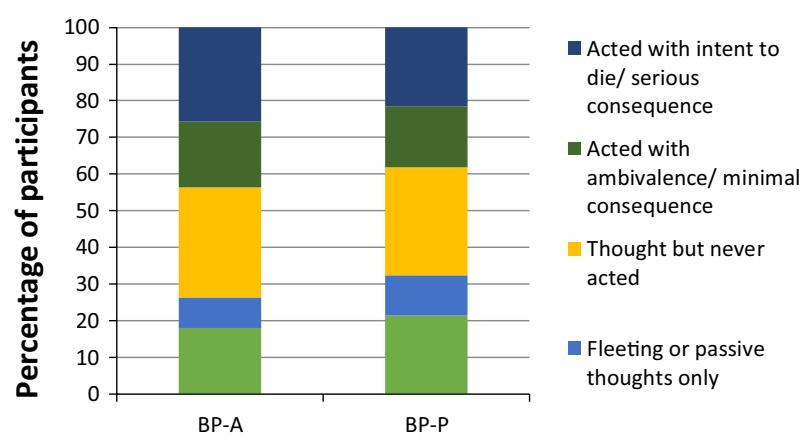

(D) Functional impact of illness

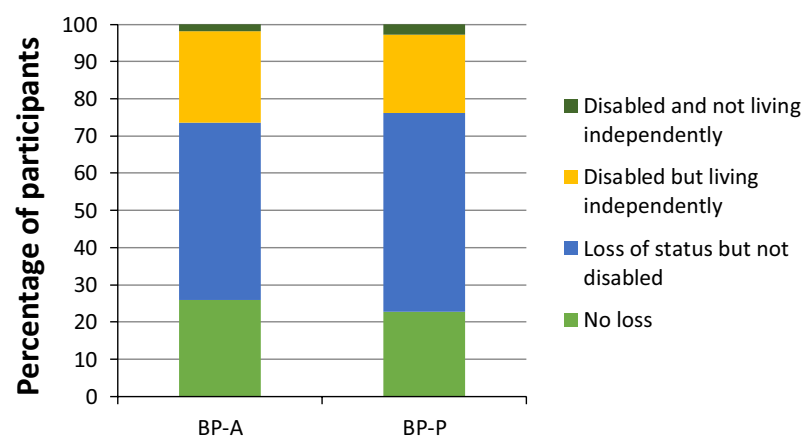

(F) Chronicity of affective disorder

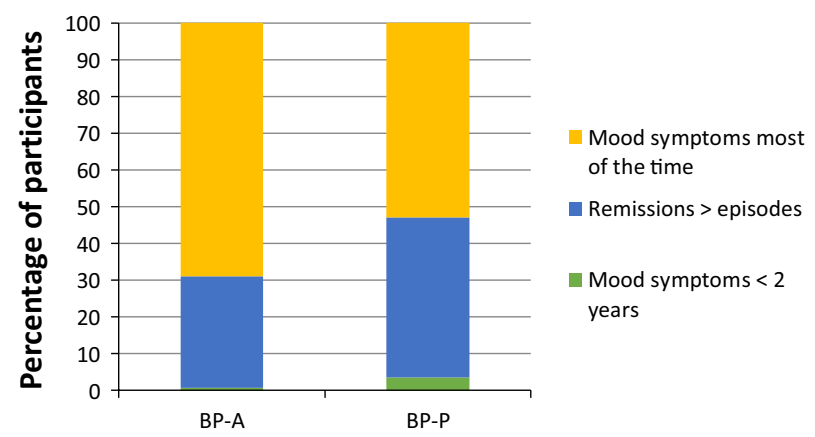

FIGURE 2 Clinical and functional comparisons between affective-only bipolar disorder (BP-A) and bipolar disorder with a history of psychosis (BP-P). (A) History of mixed episodes. (B) History of suicidality. (C) History of substance abuse. (D) Functional impact of illness. (E) First-degree relative with bipolar disorder. (F) Chronicity of affective disorder. (G) Presence of rapid cycling [Colour figure can be viewed at wileyonlinelibrary.com]

The prevalence of psychosis in this sample (53\%) is similar to other published reports. ${ }^{4,11}$ The type and content of psychosis endorsed in this sample are also consistent with the BP literature, in that a large number of participants endorsed grandiose ideation and relatively few endorsed Schneiderian first-rank symptoms that are traditionally more evident in schizophrenia. ${ }^{4}$ For the most part, psychosis in 
TAB LE 1 Demographic and clinical characteristics and comparisons between affective-only bipolar disorder (BP-A) and bipolar disorder with a history of psychosis (BP-P) participants

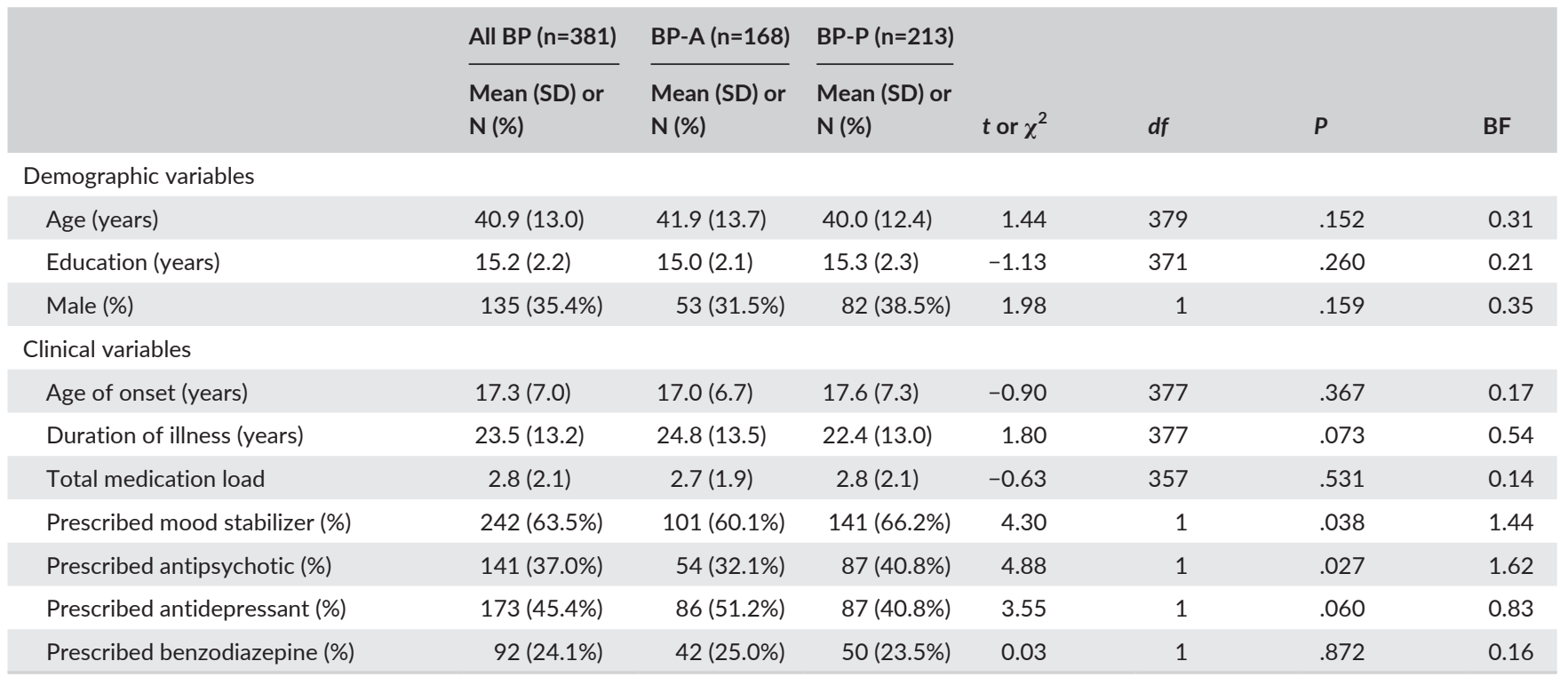

$\mathrm{BF}$, Bayes factor; SD, standard deviation.
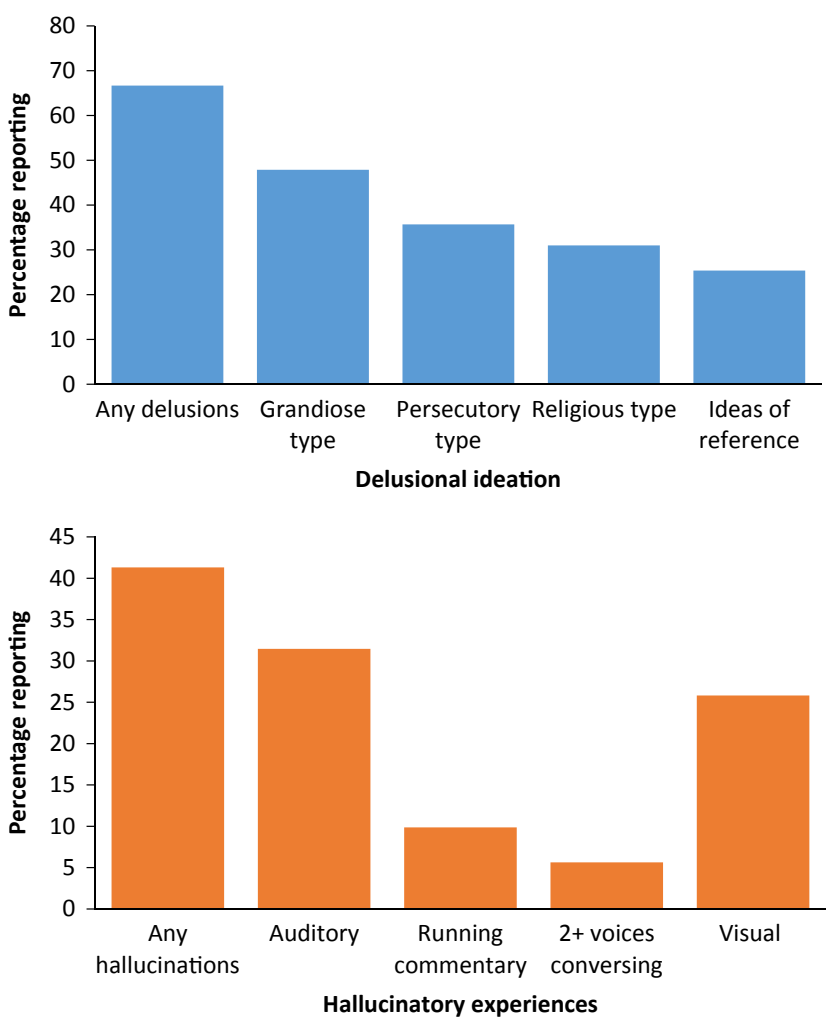

FIGURE 3 Psychosis phenomenology among bipolar disorder with a history of psychosis (BP-P) participants $(n=213)$

$\mathrm{BP}$ also tends to be mood congruent and of briefer duration than in schizophrenia. Such a qualitative difference in psychosis between BP and schizophrenia suggests that, experientially, psychotic symptoms may manifest differently in the context of a mood disorder vs a primary psychotic disorder; that is, the experience of psychosis as part of broader psychopathology may not improve diagnostic specificity, although the content may offer a clue as to the primary etiology and in turn inform appropriate treatment planning. Clinicians may find this helpful when conducting cross-sectional diagnostic interviews and attempting to achieve the difficult task of distinguishing between BP and schizophrenia-spectrum disorder.

In general, and somewhat contrary to expectation, our analyses largely failed to find significant clinical or functional differences between the groups. Consistent with Aminoff et al., ${ }^{10}$ in the full sample BP-P participants were found to have shorter duration of illness than BP-A participants. The difference of 22 vs 25 years is not likely to be clinically significant, and because participants did not differ on current age or age of onset, it could simply reflect a sampling issue (i.e., individuals experiencing psychosis may have come to the attention of referral sources more swiftly than their counterparts without psychosis). The results also indicate that BP-A participants had more chronic affective symptoms and rapid cycling, consistent with a subtype of patients with primary mood burden (e.g., chronic or persistent depression rather than brief, episodic psychosis). The lack of significant differences in numerous demographic or illness burden variables is consistent with other studies finding minimal differences between BP-A and BP-P participants. ${ }^{11}$

In terms of neuropsychological functioning, surprisingly BP-A and BP-P participants did not differ on measures of auditory and visual memory, fine motor dexterity, emotion processing, or executive functioning (verbal fluency and processing speed, processing speed with interference resolution, conceptual reasoning and setshifting, and inhibitory control). It is not the case that this sample was unusually neuropsychologically intact, as the BP participants performed more poorly than healthy controls in all measured domains ( $z$-scores $<0)$. So, although BP participants with and without psychosis were more cognitively impaired relative to control participants, they did not significantly differ from each other. That is to say, in this sample the presence of psychosis was not associated 

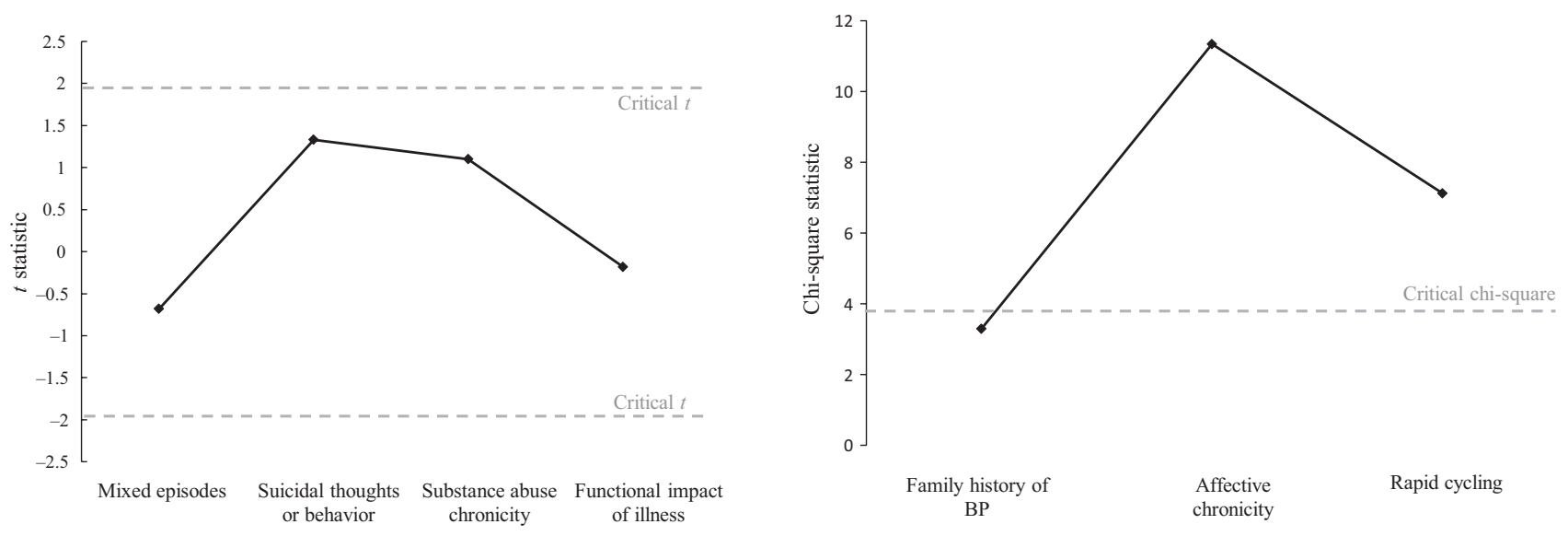

$\begin{array}{ccc}\text { Family history of } & \begin{array}{l}\text { Affective } \\ \text { chronicity }\end{array} & \text { Rapid cycling }\end{array}$

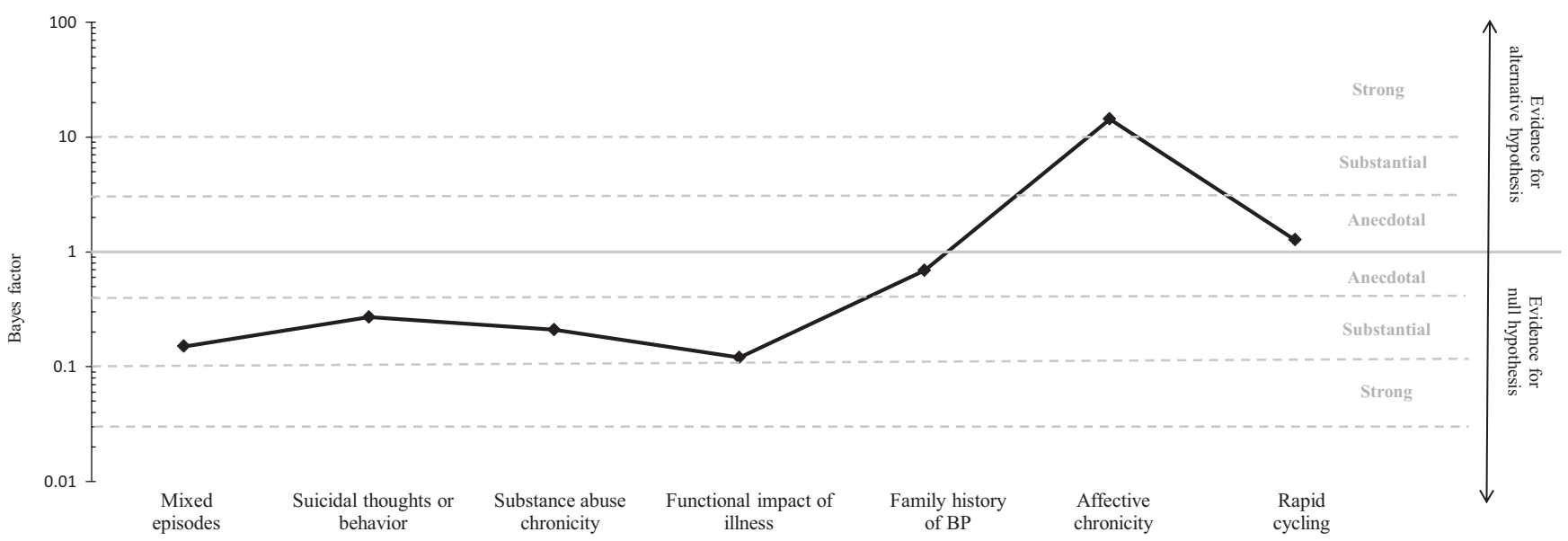

FIGURE 4 t statistics, chi-square statistics, and Bayes factors for clinical comparisons between affective-only bipolar disorder (BP-A) and bipolar disorder with a history of psychosis (BP-P).

Positive $t$ values indicate BP-A>BP-P and vice versa. The $y$-axis of the Bayes factor graph is on a log-scale

with worse neuropsychological functioning. These findings conflict with the results of Bora et al.'s meta-analysis, ${ }^{9}$ but are in line with the results of other recently published work. ${ }^{10,12,13}$ As Porter et al. point out in their review, ${ }^{19}$ mixed results between studies may reflect methodological differences in the neuropsychological domains and measures included. For example, the battery used in this study and that in the Aminoff et al. study ${ }^{10}$ and Roux et al. study ${ }^{13}$ included only the California Verbal Learning Test as a measure of verbal memory, while Bora et al. ${ }^{9}$ also included another measure of list-learning and memory, as well as two measures of story memory. Although Demmo et al. ${ }^{12}$ also included a measure of story memory and did not find significant differences, it is possible that some measures are more sensitive than others and that including more measures in a neuropsychological domain increases the ability to detect subtle differences between groups. Use of a more comprehensive and consistent neuropsychological battery in future studies (see Van Rheenen $\&$ Rossell, for example ${ }^{20}$ ) would facilitate comparisons across studies and help address the question of neuropsychological difference between BP-A and BP-P more conclusively.

This study is not without limitations, the most significant of which is that clinical information was gathered historically from retrospective self-report during a diagnostic interview and only supplemented with medical records if available. Although this limitation is inherent in any clinical research involving retrospective clinical history-taking and diagnosis, it introduces the possibility of recall bias and incomplete or inaccurate information. It is possible, therefore, that some participants were misclassified, which could cloud comparisons between groups. Longitudinal collection of clinical data (as is being done in the parent study) will be helpful to decrease reliance on retrospective report. Advanced longitudinal analyses such as trajectory analyses and longitudinal structural equation modeling are planned when the sample size of participants with 5-year data is sufficient to enable these methods to be applied. Furthermore, prospective follow-up would inform questions of whether and how psychosis in BP interacts with clinical status and neuropsychological functioning over time. Future studies focusing on participants early in the course of illness may also help reduce this bias. Moreover, whether those with BP and a history of psychosis have impaired autobiographical memory or less insight into their symptoms is deserving of future investigation, because that could also contribute to potential recall bias and inaccurate information. In addition, mood state at the time of evaluation was not formally collected or quantified, so it is unknown whether current mood state could have affected the testing results. We also did not analyze information comparing moodcongruent vs mood-incongruent psychotic features, which may have 
important implications for clinical prognosis and functional outcome; future studies would benefit from examining these features among individuals with psychotic bipolar disorder. Further, this study is limited by characteristics of the sample; for example, these participants were outpatients with relatively high educational attainment. It is not clear whether these findings would generalize to inpatient samples or those with less education or lower socioeconomic status. Finally, it would be interesting to compare these findings to those in individuals diagnosed with a primary psychotic disorder (i.e., schizoaffective disorder and schizophrenia), to enable finer grained analysis of the presence of psychosis vs the primacy of psychosis.

To our knowledge, this is the largest single study to date comparing demographic, clinical, functional, and neuropsychological features of individuals diagnosed with BP with and without psychosis. Overall, these results do not support the clinical and anecdotal notion that BP with psychotic features represents a more "severe" illness than BP without a history of psychosis. In general, the presence of psychosis does not appear to be associated with poorer clinical or functional outcomes, or suggest a greater degree of neuropsychological impairment. This is particularly intriguing in that many individuals in the BP-A group were diagnosed with BP II, which is thought to be a milder form of BP and would contribute to the expectation that BP-A participants would demonstrate less severe clinical course and neuropsychological impairment. Ultimately, although the presence of psychotic symptoms in the acute phase of bipolar illness can be distressing to patients and their families, and may require increased level of care or hospitalization for immediate management, it may not factor in to long-term prognosis or lead to more severe neuropsychological deficits as much as clinical intuition would suggest.

\section{CONFLICTS OF INTEREST}

The authors report no conflicts of interest.

\section{DISCLOSURES}

Funding sources: This work was supported by the Heinz C. Prechter Bipolar Research Fund and the Richard Tam Foundation at the University of Michigan Depression Center, the National Institutes of Health (5KL2TR000434 to I.F.T.), and the National Institute of Mental Health (K23MH108823 to I.F.T.).

\section{REFERENCES}

1. DeVylder JE, Burnette D, Yang LH. Co-occurrence of psychotic experiences and common mental health conditions across four racially and ethnically diverse population samples. Psychol Med. 2014;44:3503-3513.

2. Gaudiano BA, Zimmerman M. Prevalence of attenuated psychotic symptoms and their relationship with DSM-IV diagnoses in a general psychiatric outpatient clinic. J Clin Psychiatry. 2013;74:149-155.

3. McGrath JJ, Saha S, Al-Hamzawi A, Andrade L, Benjet C, Bromet EJ, et al. The bidirectional associations between psychotic experiences and DSM-IV mental disorders. Am J Psychiatry. 2016;173:997-1006.
4. Dunayevich E, Keck PE. Prevalence and description of psychotic features in bipolar mania. Curr Psychiatry Rep. 2000;2:286-290.

5. American Psychiatric Association. Diagnostic and Statistical Manual of Mental Disorders, 4th edn. Washington, DC: American Psychiatric Association; 1994.

6. American Psychiatric Association. Diagnostic and Statistical Manual of Mental Disorders, 5th edn. Washington, DC: American Psychiatric Association; 2013.

7. Potash JB, Willour VL, Chiu YF, Simpson SG, MacKinnon DF, Pearlson $\mathrm{GD}$, et al. The familial aggregation of psychotic symptoms in bipolar disorder pedigrees. Am J Psychiatry. 2001;158:1258-1264.

8. Potash JB, Zandi PP, Willour VL, Lan TH, Huo Y, Avramopoulos $D$, et al. Suggestive linkage to chromosomal regions $13 q 31$ and 22q12 in families with psychotic bipolar disorder. Am J Psychiatry. 2003;160:680-686.

9. Bora E, Yücel M, Pantelis C. Neurocognitive markers of psychosis in bipolar disorder: a meta-analytic study. J Affect Disord. 2010;127:1-9.

10. Aminoff SR, Hellvin T, Lagerberg TV, Berg AO, Andreassen OA, Melle I. Neurocognitive features in subgroups of bipolar disorder. Bipolar Disord. 2013;15:272-283.

11. Keck PE, McElroy SL, Rochussen Havens J, Altshuler LL, Nolen WA, Frye MA, et al. Psychosis in bipolar disorder: phenomenology and impact on morbidity and course of illness. Compr Psychiatry. 2003;44:263-269.

12. Demmo C, Lagerberg TV, Aminoff SR, Hellvin T, Kvitland LR, Simonsen $\mathrm{C}$, et al. History of psychosis and previous episodes as potential explanatory factors for neurocognitive impairment in first-treatment bipolar I disorder. Bipolar Disord. 2016;18:136-147.

13. Roux P, Raust A, Cannavo AS, Aubin V, Aouizerate B, Azorin JM, et al. Cognitive profiles in euthymic patients with bipolar disorders: results from the FACE-BD cohort. Bipolar Disord. 2017;19:146-153.

14. Nurnberger JI, Blehar MC, Kaufmann CA, York-Cooler C, Simpson SG, Harkavy-Friedman J, et al. Diagnostic interview for genetic studies: rationale, unique features, and training. Arch Gen Psychiatry. 1994;51: 849-859.

15. Langenecker SA, Saundeers EFH, Kade AM, Ransom MT, Mclnnis MG. Intermediate: cognitive phenotypes in bipolar disorder. J Affect Disord. 2010;122:285-293.

16. Ryan KA, Vederman AC, McFadden EM, Weldon AL, Kamali M, Langenecker SA, et al. Differential executive functioning performance by phase of bipolar disorder. Bipolar Disord. 2012;14:527-536.

17. Morey RD, Rouder JN. BayesFactor: computation of bayes factors for common designs. R package version 0.9.12-2 [Internet]. 2015. https://CRAN.R-project.org/package=BayesFactor.

18. Jarosz AF, Wiley J. What are the odds? A practical guide to computing and reporting Bayes Factors. J Prob Solving. 2014;7:2-9.

19. Porter RJ, Robinson LJ, Malhi GS, Gallagher P. The neurocognitive profile of mood disorders - a review of the evidence and methodological issues. Bipolar Disord. 2015;17(Suppl. 2):21-40.

20. Van Rheenen TE, Rossell SL. An empirical evaluation of the MATRICS consensus cognitive battery in bipolar disorder. BipolarDisord. 2014;16: 318-325.

21. Delis DC, Kramer JH, Kaplan E, Ober BA. Manual for the California Verbal Learning Test (CVLT-II). San Antonio, TX: The Psychological Corporation; 2000.

22. Meyers J, Meyers K. Rey Complex Figure and Recognition Trial: professional Manual. Odessa, FL: Psychological Assessment Resources; 1995.

23. Tiffin J, Asher EJ. The Purdue Pegboard: norms and studies of reliability and validity. J Applied Psychol. 1948;32:234-247.

24. Rapport LJ, Friedman SR, Tzelepis A, Van Voorhis A. Experienced emotion and affect recognition in adult attention-deficit hyperactivity disorder. Neuropsychology. 2002;16:102-110. 
25. Langenecker SA, Bieliauskas LA, Rapport LJ, Zubieta JK, Wilde EA, Berent S. Face emotion perception and executive functioning deficits in depression. J Clin Exp Neuropsychol. 2005;27:320-333.

26. Benton A, Hamsher K. Multilingual Aphasia Examination. lowa City: University of lowa; 1976.

27. Wechsler D. Wechsler Adult Intelligence Scale, 3rd edn. Cleveland: Psychological Corp; 1997.

28. Golden C. Stroop Color and Word Test. Chicago: Stoelting; 1978.

29. Reitan RM. Validity of the trailmaking test as an indication of organic brain damage. Percept Mot Skills. 1958;8:271-276.

30. Heaton RK. A Manual for the Wisconsin Card Sorting Test. Odessa, FL: Psychological Assessment Resources; 1981.
31. Langenecker SA, Caveney AF, Giordani B, Young EA, Nielson KA, Rapport $\mathrm{L}$, et al. The sensitivity and psychometric properties of a brief computer-based cognitive screening battery in a depression clinic. Psychiatry Res. 2007;152:143-154.

How to cite this article: Burton CZ, Ryan KA, Kamali M, et al. Psychosis in bipolar disorder: Does it represent a more "severe" illness?. Bipolar Disord. 2018;20:18-26.

https://doi.org/10.1111/bdi.12527 